\title{
Quantifying the Effect of Drilling Fluid Contamination on Cement-Formation Hydraulic Bond Using Scanning Electron Microscopy and Energy Dispersive Spectroscopy
}

\author{
Arome Oyibo and Mileva Radonjic \\ Craft and Hawkins Department of Petroleum Engineering, 2131 Patrick F. Taylor Hall, Louisiana State \\ University, Baton Rouge, LA 70803
}

The objective of this experimental study is to investigate the impact of physical and chemical mud contaminations on cement-formation shear bond strength for sandstone and shale formations. Physical contamination occurs when drilling fluids (mud) dehydrates on the surface of the formation, while chemical contamination on the other hand occurs when the drilling fluid (still in the liquid state) is mixed with cement slurry and reacts chemically with the cement during a cementing job. We investigated the impact of the contamination on the shear bond strength and the changes in the mineralogy of the cement at the cement-formation interface to quantify the impact of the contamination on the cement-formation shear bond strength.

Wellbore cement has been used to provide well integrity through zonal isolation in oil \& gas wells as well as geothermal wells. Cement failures could result from poor cementing, failure to completely displace the drilling fluids to failure on the path of the casing. A failed cement job could result in creation of cracks and micro annulus through which produced fluids could migrate to the surface leading to sustained casing pressure, contamination of fresh water aquifer and blow out in some cases. In addition, cement failures could risk the release of chemical substances from hydraulic fracturing into fresh water aquifer during the injection process. To achieve proper cementing, the drilling fluid should be completely displaced by the cement slurry. However, this is hard to achieve in practice, some mud is usually left on the wellbore which ends up contaminating the cement afterwards.

For this experimental study, Berea sandstone and clay rich rock discs/cores had cement bonded with them to simulate cement-formation interfaces. These interface were contaminated either physically (dehydrated clays deposited on the surface) or chemically (by intermixing drilling fluids with cement slurry). Shear bond tests were performed on the composite cores after complete hydration of cement occurred (after 28days) in order to determine the shear bond strength. Preliminary results suggested that the detrimental impact of the contamination is higher when the cores are physically contaminated i.e. when we have mud cake present at the surface of the wellbore before a cement job is performed. Also, the results showed that shear bond strength is higher for sandstone formations when compared to shale formations, implying that the low permeability formations form much weaker bond with cement. This is of particular interest to wellbore integrity issues in hydraulic fracturing where high injection pressures of fracking fluids can easily cause de-bonding of weak rock-cement interface.

Material characterization analysis was carried out to determine the micro structural changes at the cement-formation interface. Electron microscopy provided coupling of chemical/mineralogical composition with geomechanics of the interface. The phase compositions were characterised using a Jeol 8530F EPMA (with 5 wavelength dispersive spectrometres and a SDD energy dispersive spectrometer). Line transects were used to assess variations in the bulk composition. Abundances of 
phases were estimated using the Thermo NSS and Compass software on a Hitachi S3500N SEM with a energy dispersive spectrometer. 